\title{
PLANNING GRASPING MOTIONS FOR HUMANOID ROBOTS
}

\author{
JAN ROSELL, RAÚL SUÁREZ, NÉSTOR GARCÍA, MUHAYY UD DIN \\ Institute of Industrial and Control Engineering, Universitat Politècnica de Catalunya \\ Barcelona, Spain \{jan.rosell, raul.suarez, nestor.garcia.hidalgo, muhayyuddin.gillani\}@upc.edu
}

\begin{abstract}
This paper addresses the problem of obtaining the required motions for a humanoid robot to perform grasp actions, trying to mimic the coordinated hand-arm movements humans do. The first step is the data acquisition and analysis, which consists in capturing human movements while grasping several everyday objects (covering four possible grasp types), mapping them to the robot and computing the hand motion synergies for the pre-grasp and grasp phases (per grasp type). Then, the grasp and motion synthesis step is done, which consists in generating potential grasps for a given object using the four family types, and planning the motions using a bi-directional multi-goal samplingbased planner, which efficiently guides the motion planning following the synergies in a reduced search space, resulting in paths with human-like appearance. The approach has been tested in simulation, thoroughly compared with other state-of-the-art planning algorithms obtaining better results, and also implemented in a real robot.
\end{abstract}

Keywords: motion planning; grasp planning; human-like appearance

\section{Introduction}

Humanoid robots equipped with anthropomorphic dexterous hands are among the most representative examples of robots becoming more sophisticated and complex. The anthropomorphic mechanical hands are devices that concentrate in a compact volume a high number degrees of freedom (DOFs), ranging usually from 12 to 25 DOFs, as well as several different sensors. Obtaining a satisfactory performance of these hands requires the automatic planning of their movements, which is still an arduous and non-evident task since the complexity of the planning problem increases exponentially with the number of DOFs. Furthermore, sometimes not only a feasible path is required but also the one that optimizes some quality metric (e.g. minimizing the path length or the energy consumption). Regarding humanoid robots, for instance, the motion planning must not only focus on the efficient search of a valid solution, but also on the search of robot movements that mimic the motions of the human beings. In this way, the human-robot collaboration is facilitated, because humans are familiar with the robot motions and thus they can adjust their motions to avoid possible injuries or enhance the collaboration [1].

Motion planning of complex systems has been addressed using different planning algorithms, being the sampling-based planners [2] and, especially among them, the Probabilistic Roadmap planners, PRM [3], and the Rapidly-exploring Random Trees, RRT [4], the most commonly used. These algorithms have been extensively studied and, hence, several variants exist, for instance to deal with task constraints [5], to bias the sampling towards better regions of the configuration space 
by using potential fields [6] or retraction-based methods [7], or to obtain continuous and smooth trajectories satisfying geometric and dynamic constraints [8].

On the other hand, the robot joints must be properly coordinated in order to obtain human-like motions. Thereby, real human movements are commonly used as a reference [9], either pursuing a direct on-line teleoperation of the robot [10], or with the aim of analyzing these movements and getting some valuable information to be applied later in a planning phase [11]. Some relevant pioneering works dealt with the grasping problem analyzing the correlations of the finger joints when the human hand was grasping objects [12]. These correlations were called hand postural synergies and mapped into a mechanical hand [13]. The synergies existing in the human hand were also used for other objectives such as the analysis and design of robotic hands in order to mimic human grasps [14], the design of specific hand control systems $[15,16]$, or the identification of the hand pose using low-cost gloves [17]. Nevertheless, there exist other approaches that, instead of studying the hand synergies while grasping an object, compute the synergies from hand movements when the human tries to cover the whole hand configuration space in an unconstrained way $[18,19]$. More recently, a compliant model, called soft synergies, was also introduced and used in the selection of grasping forces, in their control, and in the control of the motion of the grasped object [20,21]. In addition, synergies were also used in a dual-arm anthropomorphic system while performing manipulation tasks $[11,22]$. The works mentioned above dealt with synergies involving correlations between joint positions. A more recent work extended the concept of synergies to the velocity space (i.e. the space of the first derivative of the configuration trajectories) calling them first-order synergies [23, 24] (in contrast with the synergies in the configuration space, that were called zero-order synergies).

This work proposes to characterize for the robot the synergies existing in the human grasping motions, considering different grasp types and grasp phases. Then, for a given object to be grasped, the proposed approach looks for a set of potential grasps using these synergies and uses a sampling-based motion planner, especially designed to exploit these synergies, to reduce the planning complexity and, at the same time, achieve hand-arm movements with human-like appearance. The work presented here is an extension of the work presented in [25] that includes the grasping planning phase, more detailed descriptions of the motion planning algorithms and the experimental validation of the approach.

After this introduction, Section 2 presents the problem statement and gives an overview of the proposed approach. Then, Sections 3, 4, 5 and 6 describe each of the main blocks of the work, namely, the capture of human motions, their analysis, the synthesis of grasps and the motion planning of the robot arms, with each section including experimental results that validate the proposed approach. Finally Section 7 presents the conclusions and future work. 

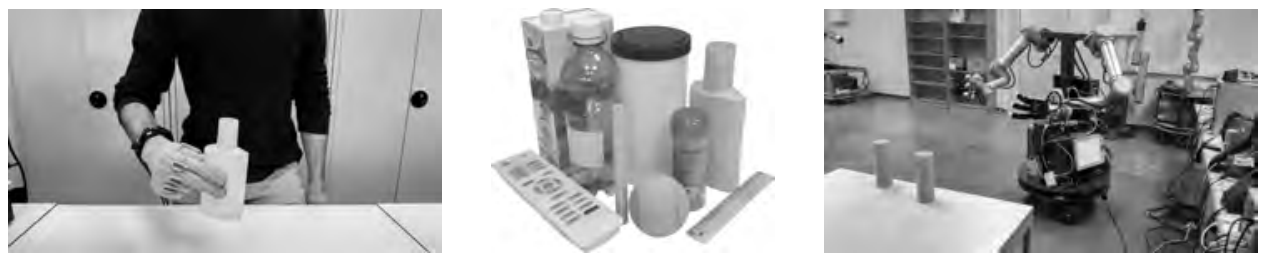

Fig. 1. Human operator wearing the measurement equipment (left), set of grasped objects in the experiments (middle), and dual-arm robot (right).

\section{Problem statement and approach overview}

The goal of this work is to plan the motions of a hand-arm robotic system trying to mimic the hand-arm movements that a human does to pick an object performing different types of grasp. To this end, a sampling-based planning algorithm is designed and the movements of a human operator are used to guide the motion planning. The main steps of the proposed approach are the following. They are grouped in two phases, a general phase to analyze the human motions when performing generic grasping operations, and an application phase devoted to sythesize both the grasp configurations and the motions required to grasp a particular object:

General analysis phase:

(1) Motion capture and mapping: The motions of a human operator performing different types of grasp on several objects are captured, as shown in Fig. 1, and then mapped to the robot whose motions are aimed to be planned (Section 3).

(2) Motion analysis: The synergies existing in the human motions when a given grasp is done are computed from the captured motions, and used to define a low-dimensional space for grasp and motion planning purposes, being this dimension-reduction process dependent on the grasp type (Section 4).

Application synthesis phase:

(3) Grasp synthesis: For a given object to be grasped, grasps of different types are computed using the corresponding synergies (Section 5).

(4) Motion planning: A bidirectional sampling-based planner is designed to use the proposed dimension-reduction method and to bias the tree growth towards the directions of the computed synergies, hence, obtaining human-like movements with a low computational load (Section 6).

Fig. 2 shows a flowchart describing the main steps and actions of the proposed approach. Each of the main blocks is detailed in a specific section below, including in each of them a subsection with a description of the theoretical framework and another one with the details of the implementation and the experimental results. 


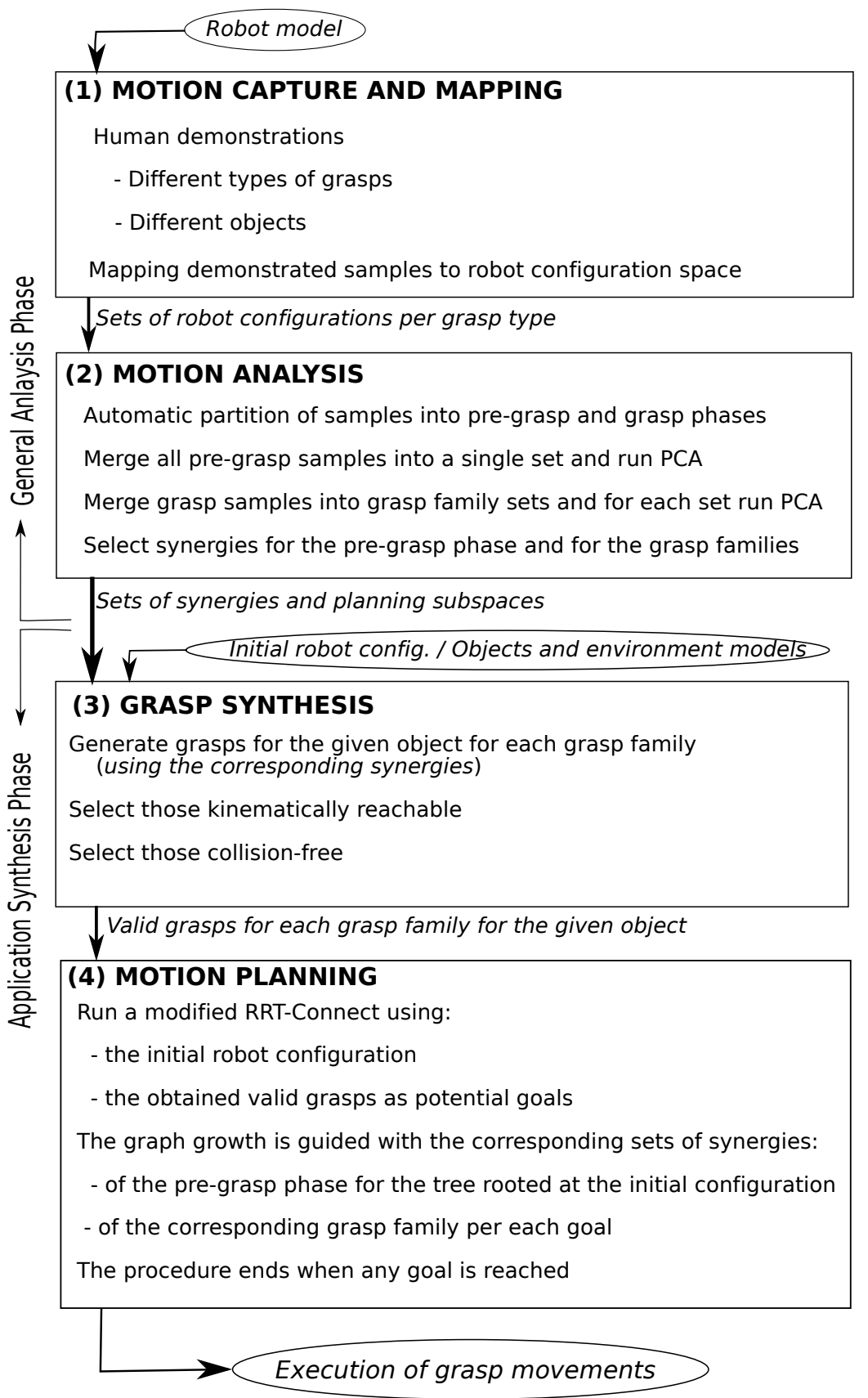

Fig. 2. Flowchart describing each step of the proposed two-phase approach. 


\section{Motion capture and mapping}

\subsection{Theoretical framework}

In this work, human motions are used as a reference to obtain human-like movements of a hand-arm robotic system picking a given object. Many types of common human grasps are gathered in the grasp taxonomy of M.R. Cutkosky [26], which classifies the grasps depending on the object size and on the type of grasp, and which was extended in [27]. Later, W. Dai, Y. Sun and X. Qian [28] updated the taxonomy of Cutkosky and analyzed, from a different perspective, the entire grasping trajectory and not only the grasping configuration (i.e. the final snapshot), proving that the grasp types can be grouped naturally into consistent grasp families (see Fig. 3). This family-grouping is used here to adapt the planning process according to the grasp being performed (even though several potential grasp types are considered simultaneously).

\subsection{Experimental results}

Using a Cyberglove sensorized glove with a $50 \mathrm{~Hz}$ sampling frequency, the motions of a human operator are recorded performing 15 different grasp types on 9 objects, with 12 repetitions per grasp type and starting off from a comfortable stand position in front of the object (see Fig. 1-left and Fig. 3). This implies 180 demonstrations and more than 15000 configuration samples, each one containing 22 measurements describing the positions of the finger joints read from the glove. Once the samples have been captured, they are mapped to the robotic hand. This mapping depends on the kinematic structure and particularities of the used robotic system. In this work, a robotic hand-arm system composed of a 6-DOF UR5 robotic arm equipped with a 16-DOF Allegro Hand is used (see Fig. 1-right). On the one hand, the information regarding the little finger is discarded and a joint-to-joint mapping is used for the flexion/extension joints of the other three fingers and the thumb. On the other hand, a fingertip-position mapping is used to compute the mapped values of the abduction/adduction joints of the fingers and the thumb, and also the value of the thumb opposition joint.

\section{Motion analysis}

\subsection{Theoretical framework}

The synergies (i.e. couplings between DOFs) are obtained running a Principal Component Analysis (PCA) over the set of hand configurations mapped from the human movements. This returns a new basis of the hand configuration space, with the axes sorted in decreasing order of the associated sample variance (i.e. the first axis marks the direction with maximum sample variance and so on). Each axis is called a synergy and the motion along it, equivalent to a single DOF, implies the movement of several (or all) joints. Although nonlinear approaches to obtain synergies have been 


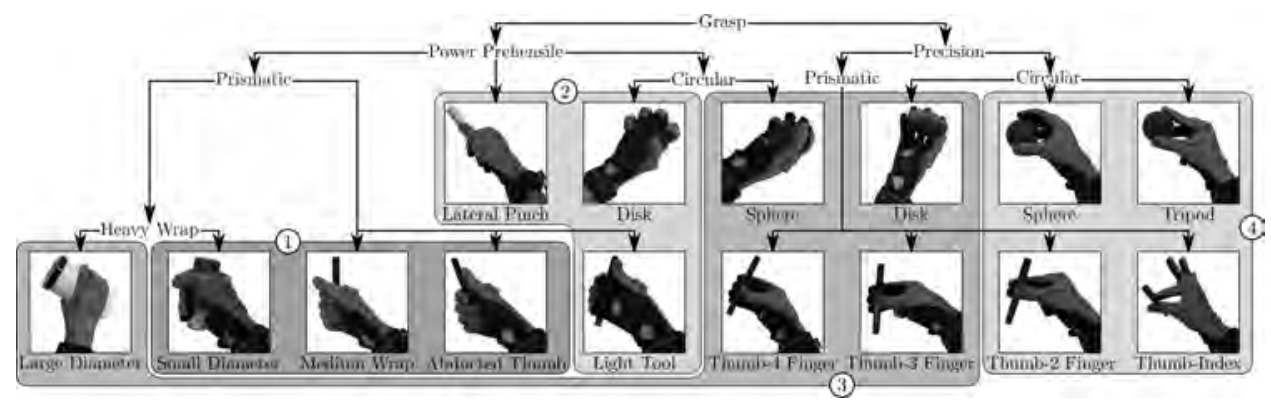

Fig. 3. The 15 force-closure grasps whose movements have been captured in this work, classified following the tree structure of the M.R. Cutkosky [26] taxonomy and grouped into grasp families, 1 to 4 , according to W. Dai, Y. Sun and X. Qian [28].

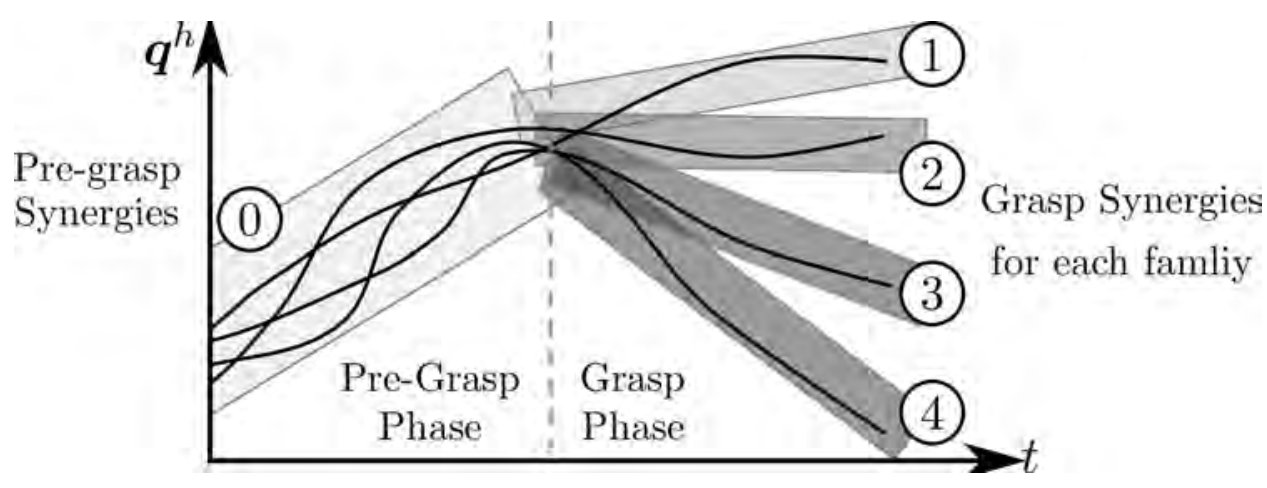

Fig. 4. Hypothetical mapped trajectories on the hand-configuration space, divided into pre-grasp and grasp phases, to obtain the common pre-grasp synergies (0) and the grasp synergies of each family (1 to 4$)$.

also proposed (e.g. [29]), the simple linear approximation of the PCA is enough to capture the subspace where the demonstrated motions lie, being useful and implementable by a drive mechanism [30] and a real-time algorithm [15].

Two phases can be observed in the mapped grasping motions (see Fig. 4). During the first phase, called pre-grasp phase, the trajectories of the hand joints are common motions opening the hand similarly in all the executions, regardless of the grasp type performed. Then, there is a certain moment in which the demonstrated trajectories begin to differ and specialize according to the type of grasp being carried out. This is the grasp phase itself. Nevertheless, the transition from one phase to the other is diffuse and does not occur at the same time for all the demonstrations. Hence, the transition time is computed as follows. Let $Q$ be the set of hand configurations mapped from a given grasping demonstration, and, for a given time instant $t$, let $Q_{t}^{-}$and $Q_{t}^{+}$be the sets of configurations in $Q$ captured before and after $t$, respectively. In addition, let the likeness of the two given sets $Q_{A}$ and $Q_{B}$ 
of hand configurations be defined as the overlapping between the distributions of the configurations in the sets, which is a measure of the similarity between $Q_{A}$ and $Q_{B}[22]$. This index can be computed as

$$
\mathcal{L}\left(Q_{A}, Q_{B}\right)=\frac{e^{-\frac{1}{2}\left(\boldsymbol{\mu}_{A}-\boldsymbol{\mu}_{B}\right)^{\top}}\left(\Sigma_{A}+\Sigma_{B}\right)^{-1}\left(\boldsymbol{\mu}_{A}-\boldsymbol{\mu}_{B}\right)}{\sqrt{(2 \pi)^{1+2 n}\left|\Sigma_{A}+\Sigma_{B}\right|}}
$$

where $n$ is the number of DOFs of the hand, $\boldsymbol{\mu}_{A}$ and $\boldsymbol{\mu}_{B}$ are the barycenters and $\Sigma_{A}$ and $\Sigma_{B}$ are the covariance matrices of the configurations in the sets $Q_{A}$ and $Q_{B}$, respectively. Then, the time instant $t$ indicating the transition between the two phases is defined as the one minimizing $\mathcal{L}\left(Q_{t}^{-}, Q_{t}^{+}\right)$.

Once the transition time between the pre-grasp and the grasp phases has been identified for each execution, all the samples in the pre-grasp phases have been grouped and used to compute the pre-grasp synergies. On the other hand, the samples in the grasp phases have been grouped according to the grasp family which each demonstrated grasp belongs to, and, then, a set of grasp synergies has been computed for each grasp family (see Fig. 3). In this way, the pre-grasp synergies explain the hand motions in the pre-grasp phase in all the grasps, and each set of grasp synergies models the hand motions of each grasp family.

For a robotic hand with $n$ DOFs, the synergies define an $n$-dimensional box centered at the barycenter of the configurations used to obtain the synergies and with each side aligned with a synergy [31]. In order for the box to contain the $(100-\alpha) \%$ of the configuration distribution for a given $\alpha$ (i.e. any hand configuration inside the box would then be similar to the ones used to compute the synergies), each side of this box is fixed to $2 \sqrt{2} \operatorname{erf}^{-1}(\sqrt[n]{1-\alpha})$ times the standard deviation of the configurations in the corresponding direction. The dimension of the box can be reduced by using only $k<n$ synergies (picking them in decreasing order) such that $k$ is the minimum value making the accumulated variance be above a confidence level of $(100-\beta) \%$ for a given desired $\beta$.

\subsection{Experimental results}

This statistical analysis was applied to the 180 mapped trajectories obtained in Subsecction 3.2, identifying each case the pre-grasp and grasp phases. Table 1 shows the accumulated sample variance obtained for the synergies corresponding to the pre-grasp and each grasp phases.

In this work, the hand has $n=16$ DOFs, and we consider $\alpha=\beta=5 \%$. Thus, the dimension $k$ of the resulting lower-dimensional boxes, called $B_{k}$, required to make the accumulated variance be above a confidence level of $95 \%$, is 4 or 5 for the grasp phase, depending on the grasp family (see bold values in Table 1), and 6 synergies are needed for the pre-grasp phase (a little bit greater, as it was expected, since the movements of all the grasp families are considered in this case).

Despite the simplification, $B_{k}$ still represent accurately the mapped hand motions. Thereby, if the planning of the hand motions is performed in the correspond- 
Table 1. Accumulated sample variance as a function of the number $k$ of chosen synergies, for the common pre-grasp phase and the grasp phase of each of the demonstrated grasp families.

\begin{tabular}{|c||c|c|c|c|c|}
\hline \multirow{2}{*}{$k$} & \multirow{2}{*}{ Pre-Grasp } & \multicolumn{5}{|c|}{ Grasp Family } \\
\cline { 3 - 6 } & & 1 & 2 & 3 & 4 \\
\hline \hline 1 & $65.575 \%$ & $79.474 \%$ & $64.234 \%$ & $63.280 \%$ & $88.568 \%$ \\
\hline 2 & $77.795 \%$ & $86.125 \%$ & $81.877 \%$ & $84.238 \%$ & $91.955 \%$ \\
\hline 3 & $84.586 \%$ & $91.442 \%$ & $88.091 \%$ & $91.428 \%$ & $94.921 \%$ \\
\hline 4 & $90.316 \%$ & $94.015 \%$ & $92.225 \%$ & $94.377 \%$ & $\mathbf{9 6 . 6 0 6} \%$ \\
\hline 5 & $93.260 \%$ & $\mathbf{9 6 . 2 2 9} \%$ & $\mathbf{9 5 . 1 0 8 \%}$ & $\mathbf{9 6 . 3 9 4} \%$ & $97.676 \%$ \\
\hline 6 & $\mathbf{9 5 . 9 9 6} \%$ & $97.665 \%$ & $96.781 \%$ & $97.664 \%$ & $98.685 \%$ \\
\hline 7 & $97.262 \%$ & $98.315 \%$ & $97.850 \%$ & $98.569 \%$ & $99.160 \%$ \\
\hline 8 & $98.165 \%$ & $98.802 \%$ & $98.834 \%$ & $99.027 \%$ & $99.449 \%$ \\
\hline 9 & $98.901 \%$ & $99.218 \%$ & $99.241 \%$ & $99.467 \%$ & $99.624 \%$ \\
\hline$\vdots$ & $\vdots$ & $\vdots$ & $\vdots$ & $\vdots$ & $\vdots$ \\
\hline 16 & $100 \%$ & $100 \%$ & $100 \%$ & $100 \%$ & $100 \%$ \\
\hline
\end{tabular}

ing $B_{k}$, the planning complexity is reduced and the obtained motions are similar to the movements mapped from the human operator.

\section{Grasp synthesis}

\subsection{Theoretical framework}

Grasp can be generated using different procedures, but a simple one is using GraspIt! [32]. It is a robotic grasping simulator that allows to obtain force-closure grasps. The following data are introduced to the simulator: a) the model of the mechanical hand of the robot, where some grasping points on the finger tips and the palm are predefined; b) the model of the object to be grasped, the model of the surface where it is placed, and the models of other obstacles nearby that may obstruct some grasp configurations; and c) the set of synergies corresponding to a given family of grasps. The simulator randomly samples a hand pose, closes the fingers following a motion obtained as a linear combination of the synergies, and checks whether the resulting grasp is force-closure or not, and gives a grasp quality measure. This is repeated a given number of times and the best grasps are returned. This procedure is encoded in the function GRAsPIT and used in Algorithm 1 where, for all the grasp families and for each grasp returned by this function, computes the inverse kinematics of the arm and, if available, verifies whether it is collision-free. Therefore, the FindGraspConfigs function detailed in Algorithm 1 returns grasp configurations that have a good grasp quality and are feasible and collision-free.

\subsection{Experimental results}

As application examples of the described procedure, Fig. 6 shows some of the best grasps obtained for a bottle placed on a shelf among other bottles, and Fig. 7 the obtained grasps for a box lying on a table among other objects. For these examples, 


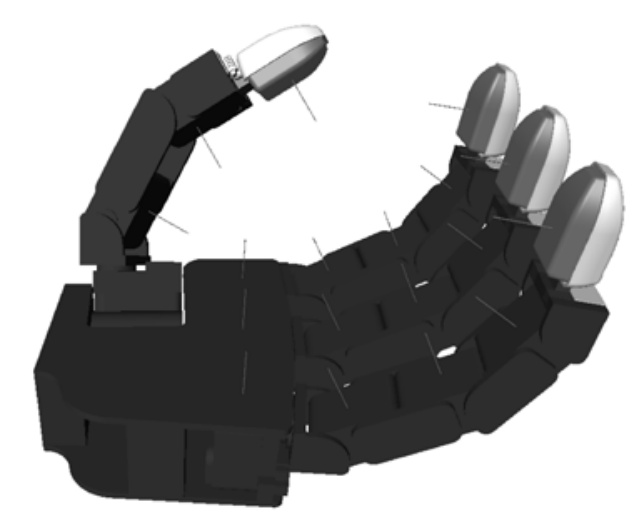

Fig. 5. Contact points on the Allegro hand used by GraspIt! to find force-closure grasps: 4 per finger, 3 for the thumb and 3 on the palm.

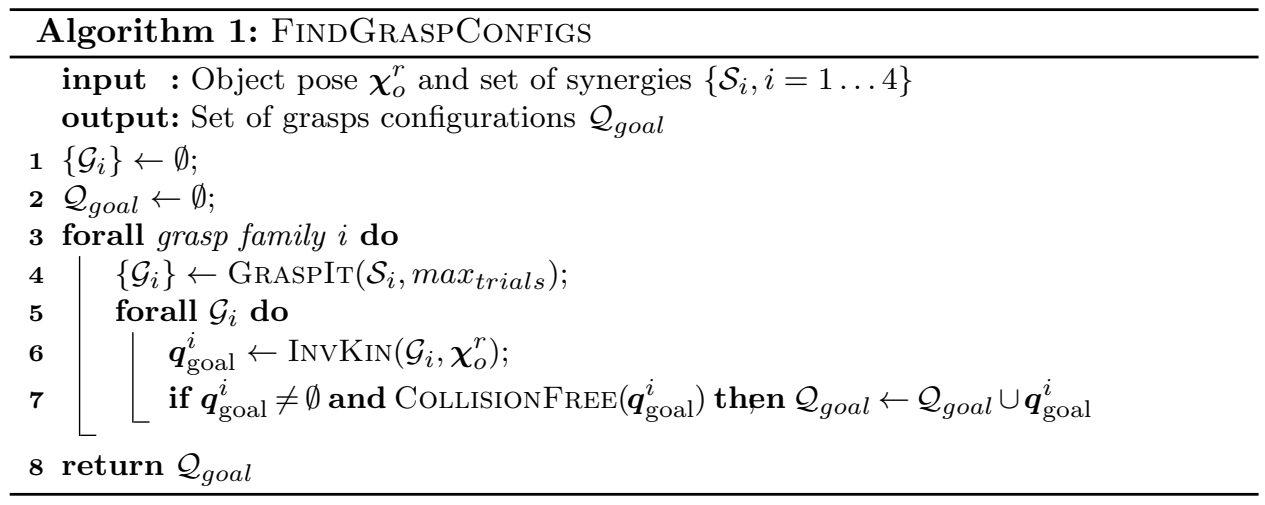

GraspIt! has been configured to test, for each grasp family type, up to 70,000 grasps and the best five are finally returned. The average time per family type was $34 \mathrm{~s}$. This time depends on the number of triangles in the meshes defining the geometries of the models (the Allegro Hand model used in these examples is composed of 205,000 triangles) and on the number of contact points used in the hand (18 contact points have been used in these examples, as shown in Fig. 5).

\section{Motion planning}

\subsection{Theoretical framework}

The planner proposed in this work is based on the RRT-Connect [33], which is widely used in motion planning since it obtains good results even on robots with a high number of DOFs and with cluttered environments. However, it has been modified here to explore the search space by following the synergies according to the type of grasp used at the goal configuration, and to allow several goals to be 

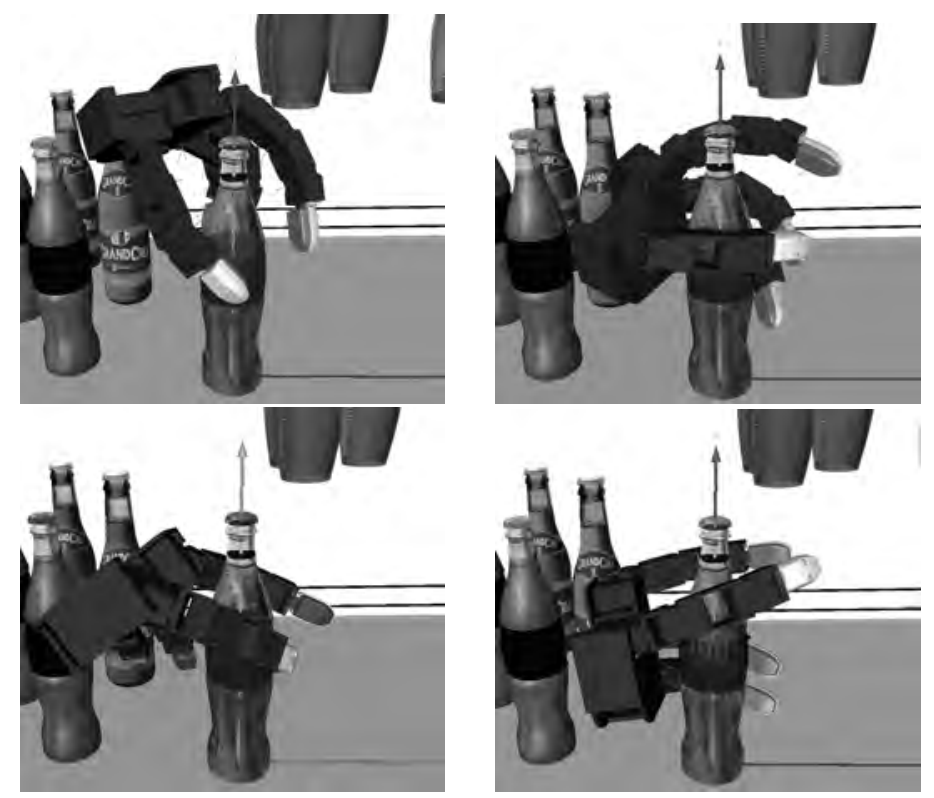

Fig. 6. Some of the grasps obtained for a bottle among obstacles.

considered simultaneouly, i.e. the changes done to the standard RRT-connect are:

a) a new extend procedure that grows the trees of samples along directions defined by the synergies,

b) a new connect procedure that tries to connect the trees of samples in a less greedy fashion, also following the synergies, and

c) a modification of the data structures and the main algorithm to allow the simultaneous growing of several trees rooted at different goal configurations, in order to deal with multigoal queries.

The proposed planner, outlined in Algorithm 2, uses the following nomenclature. Let:

- $\mathcal{C}$ be the robot configuration space defined by the joint coordinates,

- $\boldsymbol{q} \in \mathcal{C}$ be a robot configuration, defined as an arm configuration $\boldsymbol{q}^{a}$ concatenated with a hand configuration $\boldsymbol{q}^{h}$,

- $\boldsymbol{q}_{\text {start }} \in \mathcal{C}$ be a collision-free start configuration,

- $\mathcal{O}$ be the object to be grasped,

- $\chi_{o}^{r}$ be the pose of the object to be grasped, expressed with respect to the robot,

- $\mathcal{G}=\left(\boldsymbol{q}^{h}, \boldsymbol{\chi}_{h}^{o}\right)$ be a grasp, composed of the hand configuration $\boldsymbol{q}^{h}$ and the pose $\boldsymbol{\chi}_{h}^{o}$ of the hand relative to object $\mathcal{O}$ at the grasping time,

- $\left\{\mathcal{G}_{i}\right\}$ be a set of grasps for object $\mathcal{O}$. 

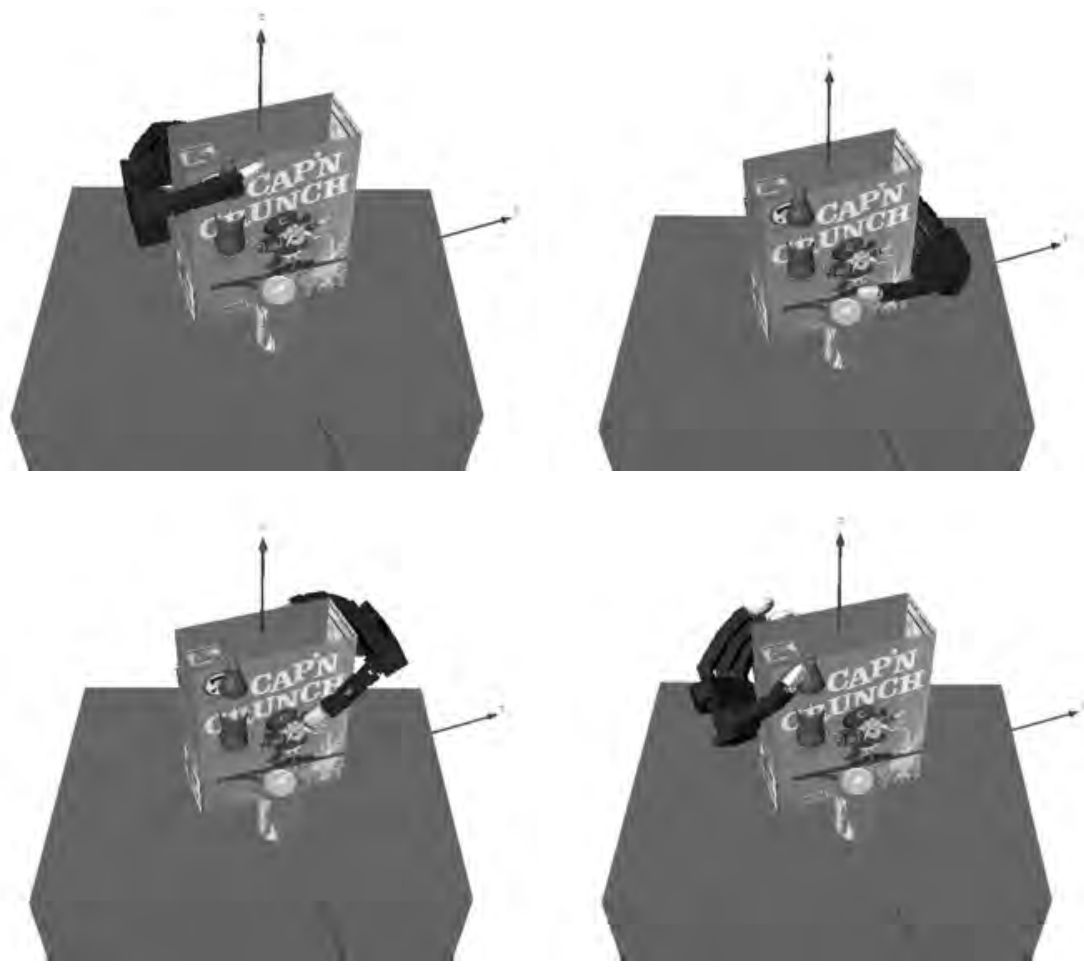

Fig. 7. Some of the grasps obtained for a box among obstacles.

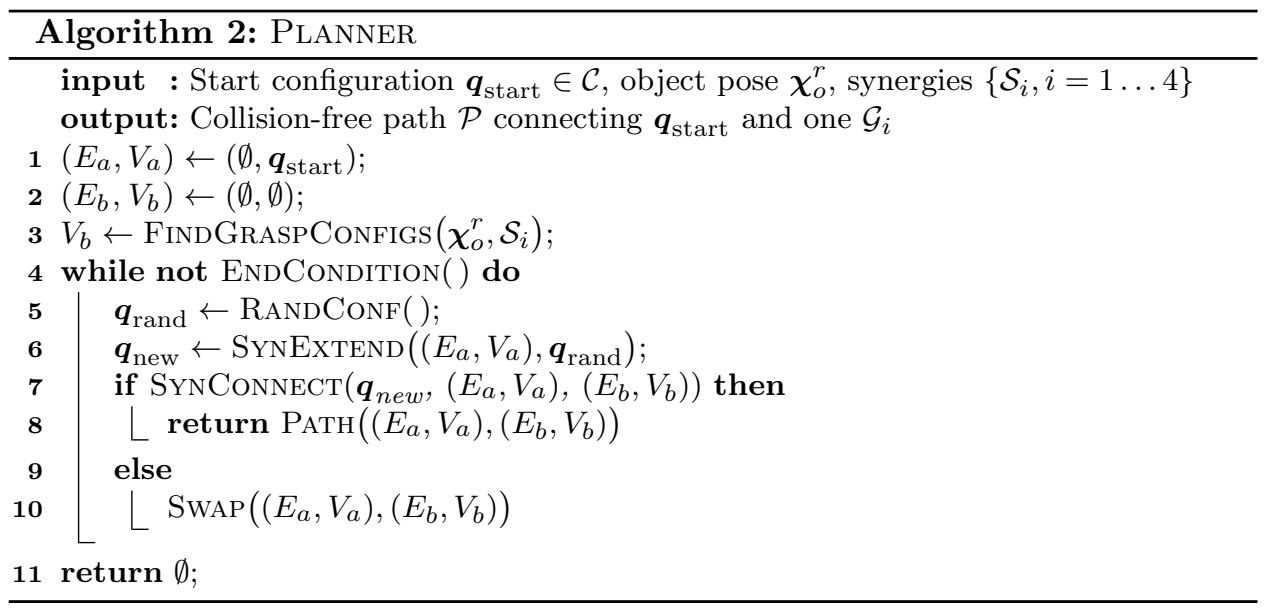

Algorithm 2 has as parameters $\boldsymbol{q}_{\text {start }}, \chi_{o}^{r}$ and $\mathcal{S}_{i}$, and returns a path from $\boldsymbol{q}_{\text {start }}$ to a goal configuration that allows to grasp the object. The planner maintains two graphs of samples, each one denoted by a pair formed by a set of edges $E$ and 


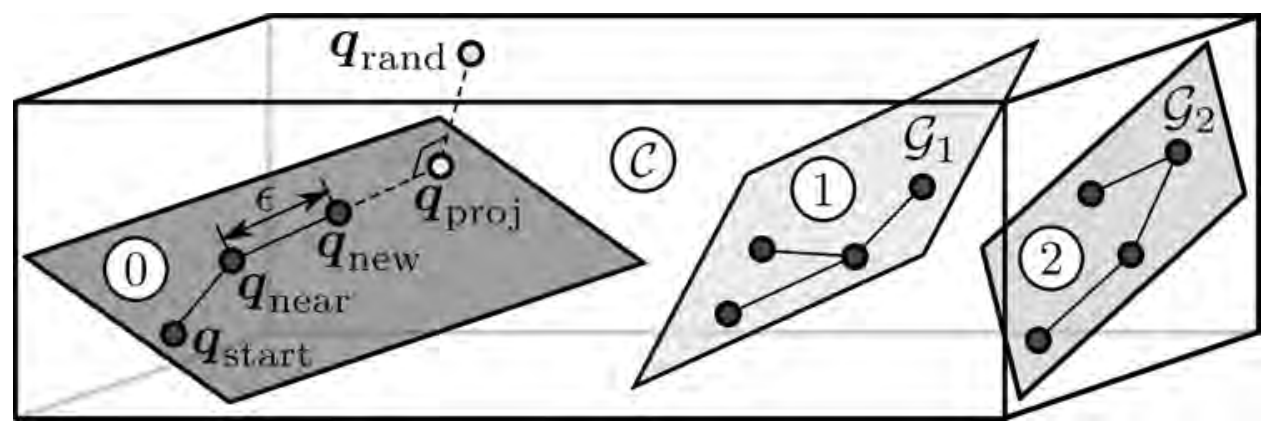

Fig. 8. Motion planning representation in $\mathcal{C}$ : sample trees rooted at the start configuration $\boldsymbol{q}_{\text {start }}$ and the grasps $\mathcal{G}_{1}$ and $\mathcal{G}_{2}$, growing close the associated synergy lower-dimensional boxes (0 to 2 ), while steering a given configuration $\boldsymbol{q}_{\text {near }}$ towards a random $\boldsymbol{q}_{\text {rand }}$ and reaching $\boldsymbol{q}_{\text {new }}$. Note that the sample trees rooted at $\mathcal{G}_{1}$ and $\mathcal{G}_{2}$ belong to the same graph structure.

a set of vertices $V$. One of this graphs represents a single tree of samples rooted at $\boldsymbol{q}_{\text {start }}$ (Line 1), and the other one contains several trees of samples rooted each one at a grasp configuration $\mathcal{G}_{i}$, computed by Algorithm 1 FindGraspConfigs explained above (Line 3), as illustrated in Fig. 8. The planner grows the trees of samples until there is a path between $\boldsymbol{q}_{\text {start }}$ and a grasp configuration. In each iteration of the process, one of the graphs is steered towards a random configuration $\boldsymbol{q}_{\text {rand }}$ (uniformly sampled in $\mathcal{C}$ ), using a variant of the basic extend method, called SynExtend (Line 6), that makes the tree grow following the synergies. A configuration $\boldsymbol{q}_{\text {new }}$ is reached if no collisions occur or $\emptyset$ is returned otherwise. Next, the connection between the graphs is greedily attempted using a variant of the basic connect method, called SynConneCt (Line 7), that also makes the tree grow following the synergies. In case the connection fails, the graphs of samples swap their roles (Line 10) and the whole process is repeated until a solution is found or some termination condition holds (Line 4), e.g. surpassing a maximum planning time, number of iterations or memory allocation. Otherwise, the solution path found is returned (Line 8). The SynExtend and SynConnect methods are explained next.

In order to integrate the synergies into the motion planning, the standard function extending the sample tree in RRT-based planners is replaced here by the function SynExtend, described in Algorithm 3. As in the classic method, a single step is performed from $\boldsymbol{q}_{\text {near }}$, the configuration in the graph closest to the desired target configuration $\boldsymbol{q}_{\text {target }}$ (Line 1), reaching a new configuration $\boldsymbol{q}_{\text {new }}$. If the rectilinear segment connecting $\boldsymbol{q}_{\text {near }}$ and $\boldsymbol{q}_{\text {new }}$ is collision-free, the segment is added to the graph and $\boldsymbol{q}_{\text {new }}$ is returned (Lines 6-8). Otherwise, $\emptyset$ is returned (Line 9). However, here, $\boldsymbol{q}_{\text {new }}$ is computed following the synergies. Thereby, if $\boldsymbol{q}_{\text {target }}$ is close enough to $\boldsymbol{q}_{\text {near }}, \boldsymbol{q}_{\text {new }}$ is set equal to $\boldsymbol{q}_{\text {target }}$ (Line 2), so that in the event that the two sample graphs are close to be connected, the guideline to follow the synergies may 

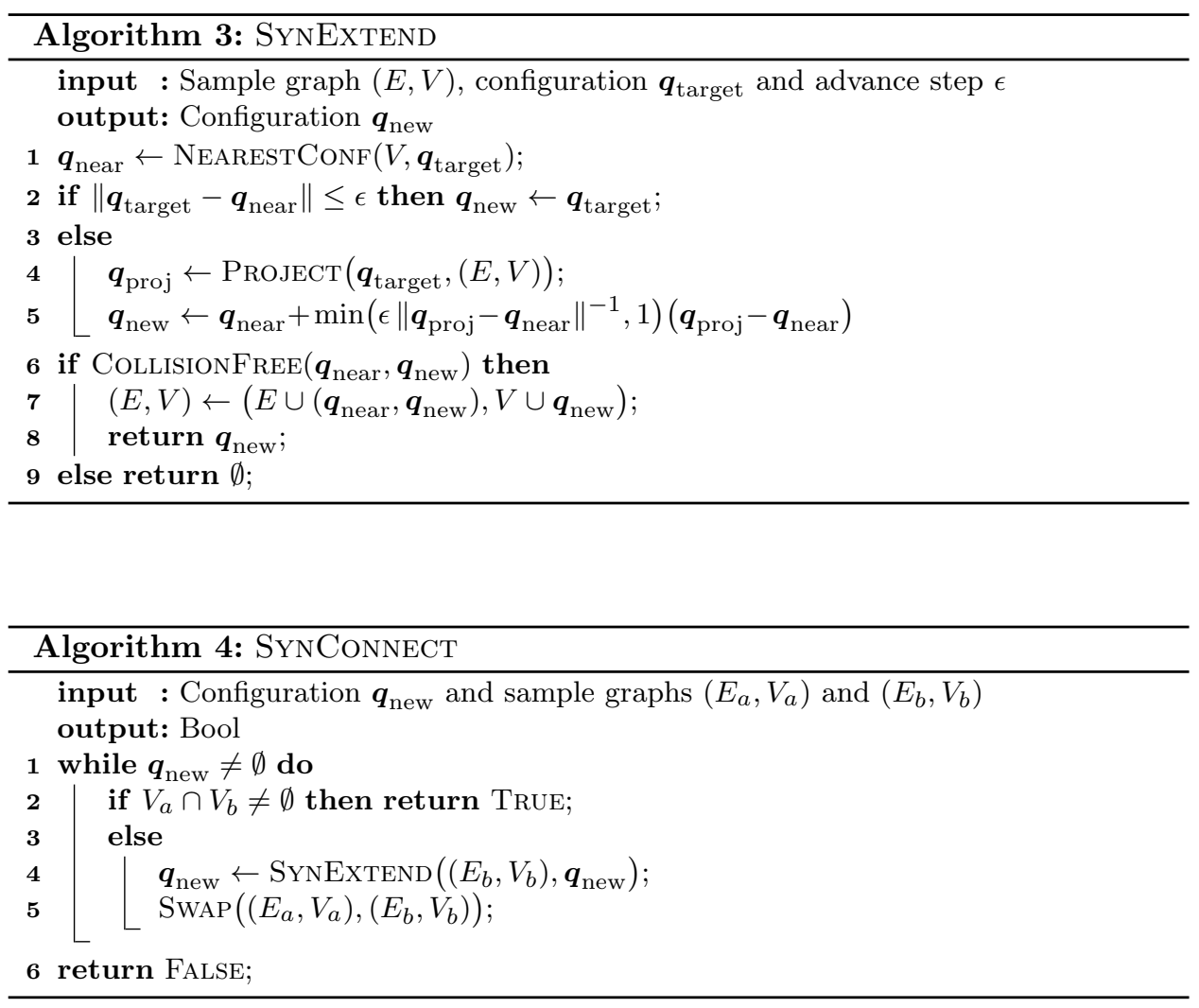

be relaxed. Otherwise, a step, with a maximum length $\epsilon$, is taken, not towards the desired $\boldsymbol{q}_{\text {target }}$ (as it would be done in the standard procedure), but towards its projection $\boldsymbol{q}_{\text {proj }}$ onto the lower-dimensional box spanned by the synergies (Lines 4-5), as illustrated in Fig. 8. The PROJECT procedure has been implemented such that the arm component of $\boldsymbol{q}_{\text {target }}$ remains the same and the hand component is projected onto the lower-dimensional box $B_{k}$ of synergies associated with the root of the sample tree containing $\boldsymbol{q}_{\text {near }}$, i.e. if $\boldsymbol{q}_{\text {near }}$ belongs to the sample tree rooted at $\boldsymbol{q}_{\text {start }}, \boldsymbol{q}_{\text {target }}$ is projected onto the box of pre-grasp synergies; otherwise, $\boldsymbol{q}_{\text {near }}$ belongs to a sample tree rooted at a certain $\mathcal{G}_{i}$ and, hence, $\boldsymbol{q}_{\text {target }}$ is projected onto the box of synergies associated with $\mathcal{G}_{i}$.

In the classic RRT-Connect, the trees of samples are connected greedily by extending one of the trees directly, along a rectilinear path in $\mathcal{C}$, until the other tree is reached or a collision occurs. However, in order to obtain a connection following the synergies, the SynCONNECT function proposed here and described in Algorithm 4 extends both graphs, in alternation and successively, using the SYNEXTEND method towards the last added configuration in the other graph (Lines 4-5), until the graphs are connected. 


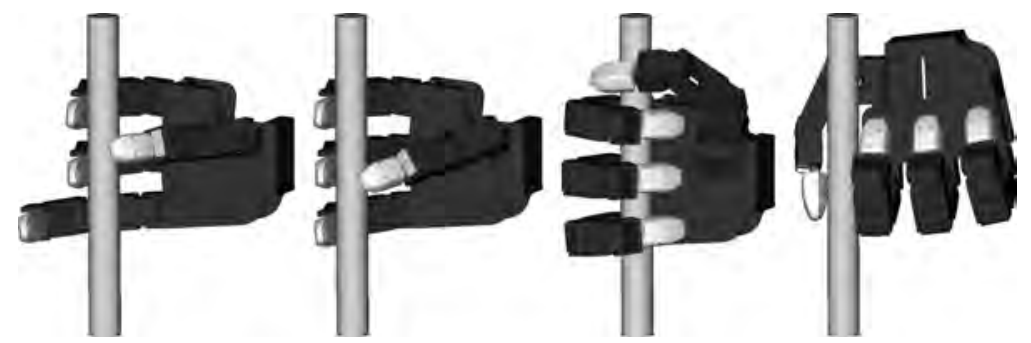

Fig. 9. Examples of grasps of each family used to grasp a cylinder: Thumb-2 Finger, Thumb-3 Finger, Medium Wrap and Lateral Pinch (from left to right, respectively).

Table 2. Average results of the motion planning when running the classic RRT-Connect (a) and the proposed approach with the proper (b) and with mismatched grasp synergies (c).

\begin{tabular}{|c||c|c|c|c|c|c|c|}
\hline $\begin{array}{c}\mho \tilde{\Xi} \\
\tilde{J}\end{array}$ & $\begin{array}{c}\text { Success } \\
\text { rate }\end{array}$ & $\begin{array}{c}\text { Planning } \\
\text { time }\end{array}$ & $\begin{array}{c}\# \\
\text { iterations }\end{array}$ & $\begin{array}{c}\text { \# collision } \\
\text { checks }\end{array}$ & $\begin{array}{c}\text { Valid } \\
\text { segments }\end{array}$ & Path length & $\begin{array}{c}\text { Human- } \\
\text { likeness }\end{array}$ \\
\hline \hline $\mathrm{a}$ & $97 \%$ & $51.80 \mathrm{~s}$ & 1834 & 32231 & $68.3 \%$ & $14.18 \mathrm{rad}$ & $73.6 \%$ \\
\hline $\mathrm{b}$ & $100 \%$ & $6.21 \mathrm{~s}$ & 274 & 10649 & $80.0 \%$ & $7.79 \mathrm{rad}$ & $83.1 \%$ \\
\hline $\mathrm{c}$ & $100 \%$ & $11.79 \mathrm{~s}$ & 484 & 13667 & $75.3 \%$ & $8.35 \mathrm{rad}$ & $81.9 \%$ \\
\hline
\end{tabular}

\subsection{Experimental results}

For illustrative purposes, the motions of an anthropomorphic dual-arm robot have been planned (see Fig. 1-right). The robot is located in front of a bookshelf and, starting off from a natural standing pose, it must grasp a cylinder standing on one of the shelves. Besides, the robot must perform human-like motions while avoiding the collisions with itself, the bookshelf and the cylinder.

In order to validate and compare the performance of the proposed approach, the following three cases have been evaluated:

a) A standard RRT-Connect, modified to tackle multi-goal queries, planning without using synergies.

b) The proposed approach, planning using the proper grasp synergies in relation of the grasp type to be performed.

c) The proposed approach, but in this case the grasp synergies and the grasp families have been intentionally mismatched (i.e. each grasp family has been randomly associated with the synergies of another grasp family).

For each execution, the planners are provided with: i) the start configuration with the robot base lightly modified at random; ii) two predefined grasps per grasp family, i.e. the automatic generation of grasps described in Section 5 has been disabled for this comparison in order to clearly illustrate the motion planning part, focusing only on it. Fig. 9 shows an example of the instances of each grasp type used to grasp the cylinder.

The experiments in each of the three cases introduced above have been imple- 


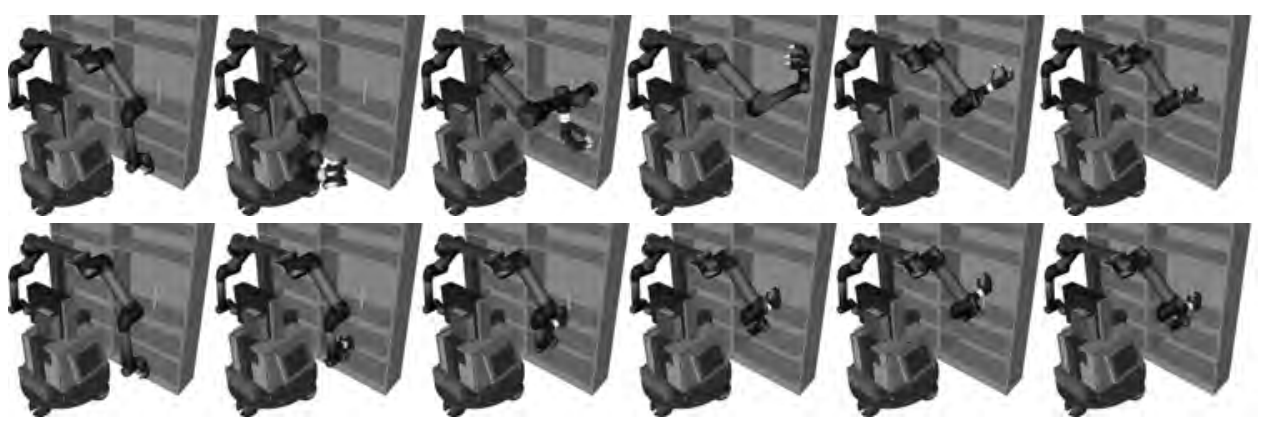

Fig. 10. Snapshots of paths obtained to grasp a cylinder from a shelf with a standard RRTConnect (top), and the proposed procedure using the proper synergies for each grasp type (bottom).

mented within the environment The Kautham Project [34], a motion planning and simulation environment developed for teaching and research purposes, and run in a 2.13-GHz Intel 2, 4-GB RAM PC. A maximum planning time of $100 \mathrm{~s}$ is considered for each planner instance. Thereby, if a path is not found within this time, the execution is marked as a failure. Fig. 10 shows snapshots of cases a and b. After 100 executions, Table 2 shows the average values of the success rate, the planning time, the number of iterations and collision checks, the rate of valid segments (i.e. the ratio of iterations in which the sample trees actually grow), the path length (measured in $\mathcal{C}$ as the weighted sum of accumulated joint movements along the path), and the path human-likeness computed as the misalignment of a path with respect to a given set of reference human movements [31]. Here, natural free-movements of the operator while moving freely the fingers in an unconstrained way (i.e. without performing any specific task), trying to cover the whole hand workspace, are used as reference of natural human movements.

Looking to the results in Table 2, it can be seen that the proposed planning approach is several times faster than the standard RRT-Connect algorithm (up to an order of magnitude). In fact, the motion planning can be solved within the time restrictions for the $100 \%$ of the executions only when the proposed approach is used, either when the grasp synergies are properly associated with the selected grasps or when they are mismatched, i.e. cases $b$ and $c$. It can be stated that the use of synergies clearly reduces the planning time since the solution is enforced to lie close to the lower-dimensional boxes $B_{k}$. This focuses the search efforts close to the demonstrated movements (which are feasible solutions), thus accelerating the connection of the sample trees and, thereby, reducing the needed number of iterations and collision checks to find a solution. In addition, since the grasp synergies are obtained from feasible movements, the probability of obtaining collision-free robot configurations increases when using synergies (see valid segments rate in Table 2), reducing greatly the computation time. The results also show that even when the 

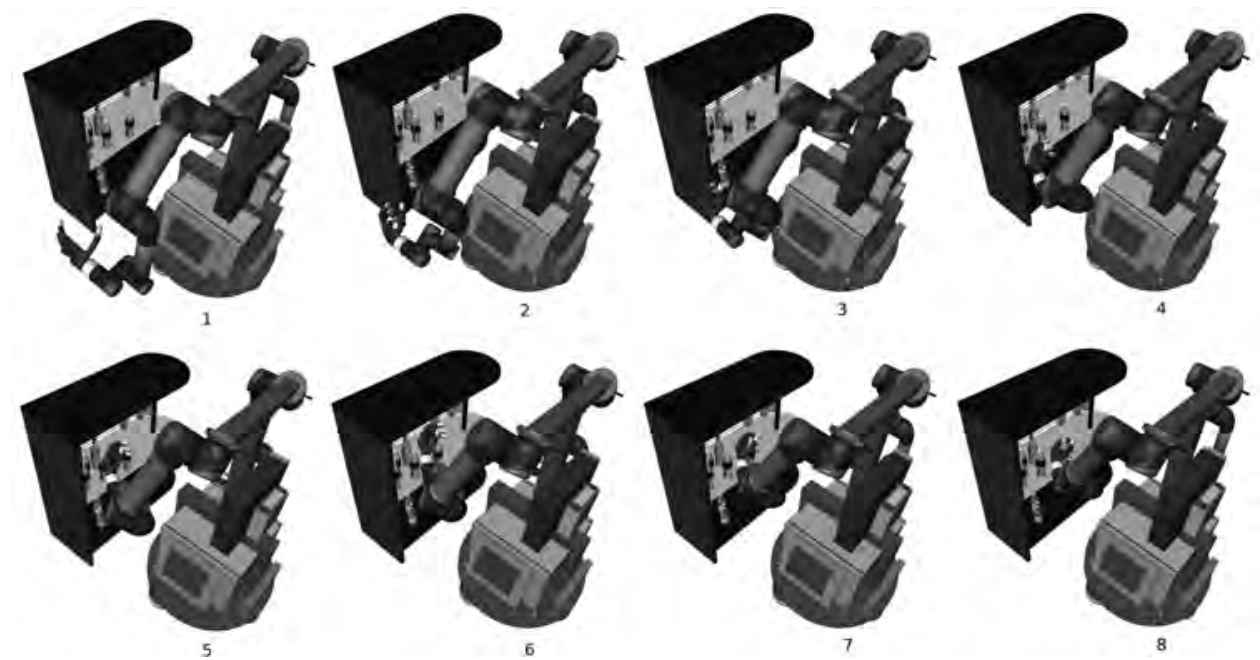

Fig. 11. Snapshots of the grasping of a bottle in a simulated environment.

correct grasping synergies are not used, i.e. case $c$, the benefits of using synergies are still evident. In this case, the planning time is slightly penalized, however, it is still a better option than not using synergies at all. On the other hand, the proposed planning procedure produces movements of the robotic system that look more natural and human-like (see human-likeness in Table 2), since the grasp synergies are obtained from human demonstrations and the human-likeness is preserved within the planning process.

Including now the grasp synthesis described in Section 5, Fig. 11 shows snapshots of the simulated final path obtained using the grasps shown in Fig. 6 for the task of grasping a specific bottle on a shelf among other bottles acting as potential obstacles, and Fig. 12 shows snapshots of the simulated path and real final execution of the task of grasping a box lying on a table in the middle of other obstacles using the grasp shown in Fig. 7. A video of the simulated and experimental validation examples is available at https://sir.upc.edu/projects/kautham/videos/IJHR.mp4.

\section{Conclusions and Future Work}

This paper has proposed a procedure to efficiently obtain human-like hand-arm movements to grasp a given object, including the generation of the grasps. To this end, the movements of a human operator performing different grasps on different objects have been captured and mapped to the robot. These grasp movements have been classified according to a grasp taxonomy, and for each grasp family a set of human-demonstrated synergies (couplings between DOFs) have been computed. In addition, a pre-grasp set of synergies has also been computed, common for all the grasp families. Finally, a motion planner profiting from these synergies has 

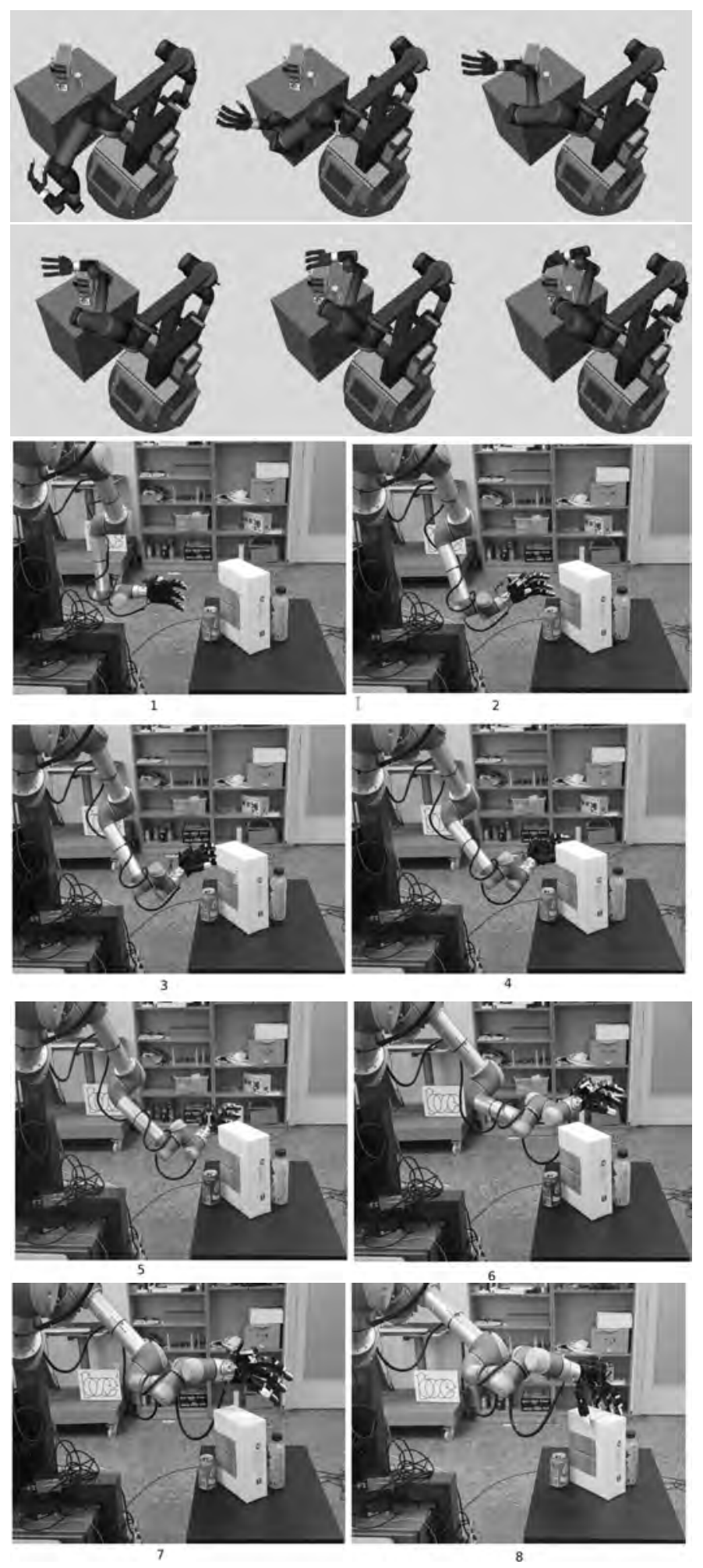

Fig. 12. Snapshots of the grasping of a box in simulation and in the real environment. 
been presented and compared against other state-of-the-art planners planning the motions of a real anthropomorphic dual-arm robot. The effect of using the grasp synergies is positive since the results improve even in the case of using synergies obtained for one type of grasp in the planning of movements for another type of grasp.

The proposal opens several interesting potential research lines, such as its extension to the velocity space and the coordination of the robot base, arms and hands all at the same time.

\section{Acknowledgements}

This work was partially supported by the Spanish Government through the project DPI2016-80077-R.

\section{References}

1. T. Fukuda, R. Michelini, V. Potkonjak, S. Tzafestas, K. Valavanis, and M. Vukobratovic. How far away is "artificial man". IEEE Robotics and Automation Mag., 8(1):66-73, Mar. 2001.

2. M. Elbanhawi and M. Simic. Sampling-based robot motion planning: A review. IEEE Access, 2:56-77, Jan. 2014.

3. L. E. Kavraki, P. Svestka, J.-C. Latombe, and M. H. Overmars. Probabilistic roadmaps for path planning in high-dimensional configuration spaces. IEEE Trans. Robotics and Autom., 12(4):566-580, Aug. 1996.

4. J. J. Kuffner and S. LaValle. RRT-Connect: An efficient approach to single-query path planning. In Proc. IEEE Int. Conf. Robotics and Automation, pages 995-1001, Apr. 2000 .

5. M. Stilman. Global manipulation planning in robot joint space with task constraints. IEEE Trans. Robotics, 26(3):576-584, June 2010.

6. N. García, R. Suárez, and J. Rosell. HG-RRT*: Human-Guided optimal random trees for motion planning. In Proc. IEEE Int. Conf. Emerging Technologies and Factory Automation, Sept. 2015.

7. J. Lee, O. Kwon, L. Zhang, and S. E. Yoon. A selective retraction-based RRT planner for various environments. IEEE Trans. Robotics, 30(4):1002-1011, Aug. 2014.

8. T. Nishi and T. Sugihara. Motion Planning of a Humanoid Robot in a Complex Environment Using RRT and Spatiotemporal Post-Processing Techniques. In International Journal of Humanoid Robotics 11(2), 2014.

9. B. D. Argall, S. Chernova, M. Veloso, and B. Browning. A survey of robot learning from demonstration. Robotics and Auton. Systems, 57(5):469-483, May 2009.

10. L. Colasanto, R. Suárez, and J. Rosell. Hybrid mapping for the assistance of teleoperated grasping tasks. IEEE Trans. Systems, Man and Cybernetics, 43(2):390-401, 2013.

11. R. Suárez, J. Rosell, and N. García. Using synergies in dual-arm manipulation tasks. In Proc. IEEE Int. Conf. Robotics and Automation, pages 5655-5661, May 2015.

12. M. Santello, M. Flanders, and J. F. Soechting. Patterns of hand motion during grasping and the influence of sensory guidance. J. Neuroscience, 22(4):1426-1435, Feb. 2002 .

13. M.T. Ciocarlie and P.K. Allen. Hand posture subspaces for dexterous robotic grasping. Int. J. Robotics Research, 28(7):851-867, July 2009. 
14. F. Ficuciello, G. Palli, C. Melchiorri, and B. Siciliano. Postural synergies of the UB Hand IV for human-like grasping. Robotics and Auton. Systems, 62(4):515-527, Apr. 2014.

15. T. Wimböck, B. Jan, and G. Hirzinger. Synergy-level impedance control for a multifingered hand. In Proc. IEEE/RSJ Int. Conf. Intelligent Robots and Systems, pages 973-979, Sept. 2011.

16. S. Li, J. He, X. Sheng, H. Liu and X. Zhu, Synergy-Driven Myoelectric Control for EMG-Based Prosthetic Manipulation: A Case Study, In International Journal of Humanoid Robotics 11(2), 2014.

17. M. Bianchi, P. Salaris, and A. Bicchi. Synergy-based hand pose sensing: Reconstruction enhancement. Int. J. Robotics Research, 32(4):396-406, Apr. 2013.

18. S. Sun, C. Rosales, and R. Suárez. Study of coordinated motions of the human hand for robotic applications. In Proc. IEEE Int. Conf. Information and Automation, pages 776-781, June 2010.

19. J. Rosell, R. Suárez, C. Rosales, and A. Pérez. Autonomous motion planning of a hand-arm robotic system based on captured human-like hand postures. Autonomous Robots, 31(1):87-102, May 2011.

20. M. Gabiccini, A. Bicchi, D. Prattichizzo, and M. Malvezzi. On the role of hand synergies in the optimal choice of grasping forces. Autonomous Robots, 31:235-252, July 2011.

21. D. Prattichizzo, M. Malvezzi, and A. Bicchi M. Gabiccini. On motion and force controllability of precision grasps with hands actuated by soft synergies. IEEE Trans. Robotics, 29(6):1440-1456, Dec. 2013.

22. N. García, R. Suárez, and J. Rosell. Task-Dependent synergies for motion planning of an anthropomorphic dual-arm system. IEEE Trans. Robotics, 33(3):756-764, June 2017.

23. N. García, J. Rosell, and R. Suárez. Motion planning using first-order synergies. In Proc. IEEE/RSJ Int. Conf. Intelligent Robots and Systems, pages 2058-2063, Sept. 2015.

24. N. García, R. Suárez, and J. Rosell. First-Order synergies for motion planning of anthropomorphic dual-arm robots. In Proc. IFAC World Congress, pages 2283-2290, July 2017.

25. N. García, R. Suárez, and J. Rosell. Planning hand-arm grasping motions with humanlike appearance. In 2018 IEEE/RSJ International Conference on Intelligent Robots and Systems (IROS), pages 3517-3522, Oct 2018.

26. M. R. Cutkosky. On grasp choice, grasp models, and the design of hands for manufacturing tasks. IEEE Trans. Robotics and Automation, 5(3):269-279, June 1989.

27. T. Feix, J. Romero, H. B. Schmiedmayer, A. M. Dollar, and D. Kragic. The GRASP taxonomy of human grasp types. IEEE Trans. Human-Machine Systems, 46(1):66-77, Feb. 2016.

28. W. Dai, Y. Sun, and X. Qian. Functional analysis of grasping motion. In $I E E E / R S J$ Int. Conf. Intelligent Robots and Systems, pages 3507-3513, Nov. 2013.

29. J. Romero, T. Feix, C.H. Ek, H. Kjellström, and D. Kragic. Extracting postural synergies for robotic grasping. IEEE Trans. Robotics, 29(6):1342-1352, Dec. 2013.

30. W. Chen, C. Xiong, and S. Yue. Mechanical Implementation of Kinematic Synergy for Continual Grasping Generation of Anthropomorphic Hand. IEEE/ASME Trans. Mechatronics, 20(3):1249-1263, June 2015.

31. N. García, J. Rosell, and R. Suárez. Motion planning by demonstration with humanlikeness evaluation for dual-arm robots. IEEE Trans. Systems, Man, and Cybernetics: Systems, PP(99):1-10, 2017. 
20 Jan Rosell, Raúl Suárez, Néstor García and Muhayy Ud Din

32. A. T. Miller and P. K. Allen. Graspit! a versatile simulator for robotic grasping. IEEE Robotics Automation Magazine, 11(4):110-122, Dec 2004.

33. J. J. Kuffner and S. LaValle. RRT-Connect: An efficient approach to single-query path planning. In Proc. IEEE Int. Conf. Robotics and Automation, pages 995-1001, Apr. 2000.

34. J. Rosell, A. Pérez, A. Aliakbar, Muhayyuddin, L. Palomo, and N. García. The Kautham project: A teaching and research tool for robot motion planning. In Proc. IEEE Int. Conf. Emerging Technologies and Factory Automation, Sept. 2014. 

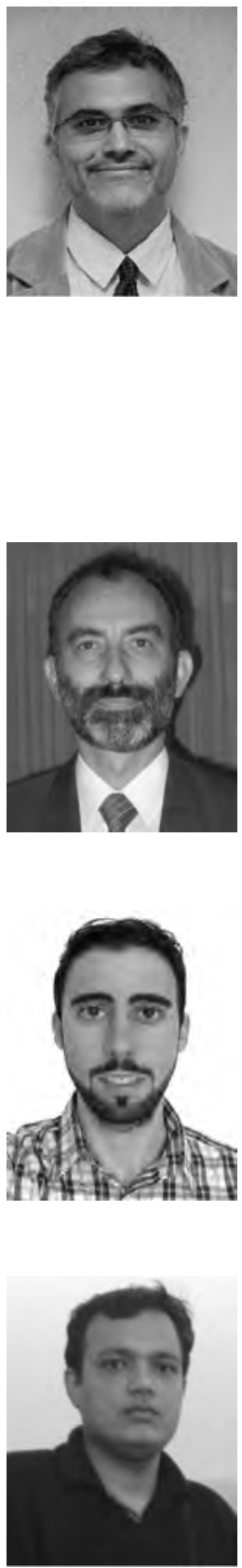

Jan Rosell received the BS degree in Telecomumunication Engineering and the Ph.D. degree in Advanced Automation and Robotics from the Universitat Politècnica de Catalunya (UPC), Barcelona, Spain, in 1989 and 1998, respectively. He joined the Institute of Industrial and Control Engineering (IOC) in 1992 where he has developed research activities in robotics. He has been involved in teaching activities in Automatic Control and Robotics as Assistant Professor since 1996 and as Associate Professor since 2001. His current technical areas include task and motion planning, mobile manipulation, and robot co-workers.

Raúl Suárez received the Electronic Engineer degree (with honors) from the National University of San Juan, Argentina, in 1984, and the Ph.D. degree (cum laude) from the Universitat Politècnica de Catalunya (UPC), Barcelona, Spain, in 1993. He is Research Supervisor at the Institute of Industrial and Control Engineering (IOC), UPC, where he has been responsible for the research line on Process Control (1998-2003), Assistant Director (2003-2009) and Director (2009-2016) and the Coordinator of the Doctoral Program on Robotics (since 1995). His main research areas include grasping and manipulation, mechanical hands, fixturing, assembly, task planning, telemanipulation and manufacturing automation.

Néstor Garcíla received the B.S. degree (with honors) in Industrial Engineering from the Universitat Politècnica de Catalunya (UPC), Barcelona, Spain, in 2015, and the Ph.D. degree in Automatic Control, Robotics and Computer Vision at the Institute of Industrial and Control Engineering (IOC), UPC, in 2019. His current research interests are focused upon task and motion planning, human-robot interaction, multirobot cooperation and learning in robotic systems.

Muhayy Ud Din received the MS degree in Computer Science from GC University Lahore, Pakistan in 2011 and Ph.D. degree in Automatic Control, Robotics and Computer Vision, from the Institute of Industrial and Control Engineering, Universitat Politècnica de Catalunya (UPC), Barcelona Spain in 2018. His current research area includes machine learning for grasping and manipulation, physics-based motion planning, mobile manipulation, and robotic software development. 